\title{
Uncertainty Management of Dynamic Tariff Method for Congestion Management in Distribution Networks
}

\author{
Huang, Shaojun; Wu, Qiuwei; Cheng, Lin; Liu, Zhaoxi; Zhao, Haoran
}

Published in:

IEEE Transactions on Power Systems

Link to article, DOI:

10.1109/TPWRS.2015.2408439

Publication date:

2016

Document Version

Peer reviewed version

Link back to DTU Orbit

Citation (APA):

Huang, S., Wu, Q., Cheng, L., Liu, Z., \& Zhao, H. (2016). Uncertainty Management of Dynamic Tariff Method for Congestion Management in Distribution Networks. IEEE Transactions on Power Systems, 31(6), 4340-4347. https://doi.org/10.1109/TPWRS.2015.2408439

\section{General rights}

Copyright and moral rights for the publications made accessible in the public portal are retained by the authors and/or other copyright owners and it is a condition of accessing publications that users recognise and abide by the legal requirements associated with these rights.

- Users may download and print one copy of any publication from the public portal for the purpose of private study or research.

- You may not further distribute the material or use it for any profit-making activity or commercial gain

- You may freely distribute the URL identifying the publication in the public portal 


\title{
Uncertainty Management of Dynamic Tariff Method for Congestion Management in Distri- bution Networks
}

\author{
Shaojun Huang, Student Member, IEEE, Qiuwei Wu, Senior Member, IEEE, Lin Cheng, Senior \\ Member, IEEE, Zhaoxi Liu, Student Member, IEEE, Haoran Zhao
}

\begin{abstract}
The dynamic tariff (DT) method is designed for the distribution system operator (DSO) to alleviate congestions that might occur in a distribution network with high penetration of distributed energy resources (DERs). Uncertainty management is required for the decentralized DT method because the DT is determined based on optimal day-ahead energy planning with forecasted parameters such as day-ahead energy prices and energy needs which might be different from the parameters used by aggregators. The uncertainty management is to quantify and mitigate the risk of the congestion when employing the DT method, which is achieved by firstly formulating the problem as a chance constrained two-level optimization and then solving the problem through an iterative procedure. Two case studies were conducted to demonstrate the efficacy of the uncertainty management of DT method.
\end{abstract}

Index Terms-- Chance constrained program, congestion management, distribution system operator (DSO), distributed energy resources (DERs), uncertainty management.

\section{INTRODUCTION}

A $S$ the penetration level of distributed energy resources (DERs), such as photovoltaic systems (PV systems), wind power systems (WPS), electric vehicles (EV) and heat pumps (HP), in distribution networks is constantly increasing, the operation of distribution networks becomes more and more challenging. One of the big challenges of the distribution system operator (DSO) is the potential congestion problems, envisaged as voltage problems (bus voltage is close to or exceeding the limit, typically $+/-10 \%$ ) and overloading problems (loading is close to or exceeding the thermal limit of power components).

The congestions in distribution networks may be caused by simultaneous charging or discharging of EVs. There are a number of congestion management methods to resolve the problems without reinforcing the distribution network. The congestion management methods can be categorized into two groups, direct control methods [1], [2] and indirect control

The work is supported by the EU Seventh Framework Programme (FP7) through the 'Ideal Grid for All (IDE4L)' project.

S. Huang, Q. Wu, Z. Liu and H. Zhao are with the Center for Electric Powe and Energy (CEE), Department of Electrical Engineering, Technical University of Denmark (DTU), 2800 Kgs. Lyngby, Denmark (e-mail: shuang@elektro.dtu.dk; qw@elektro.dtu.dk; zhliu@elektro.dtu.dk, hzhao@elektro.dtu.dk).

L. Cheng is with Department of Electrical Engineering, Tsinghua University, Beijing 100084, China (e-mail: chenglin@mail.tsinghua.edu.cn). methods, namely market based methods [3]-[6]. Market based methods have drawn a lot of attention of researchers as well as the DSOs because they have the least impact on customers and can benefit the stakeholders in the market through energy planning or other operation managements.

A recently introduced interesting market based method is the dynamic tariff (DT) method [7]-[9]. Though the DT method shares many similar features of the distribution locational marginal pricing (DLMP) method [5], [6], e.g. both methods employ optimization tools and marginal cost concept (Lagrange multipliers) and DT is equal to the congestion cost element of the DLMP, there are differences between them. First of all, the DT method is not a market clearing method while the DLMP is. The DT method relies on the existing day-ahead market, e.g. the spot market in Nordic area, and it can be seamlessly integrated into the existing market [7]-[9]. Second of all, the DT method is a decentralized control method, which is implemented through two steps of optimizations, while the DLMP is a centralized clearing method through one step of optimization.

However, the uncertainty within the DT framework and the corresponding optimization under uncertainty has not been studied previously. The uncertainty comes from the following aspects. Firstly, the uncertainty lies in the nature of the decentralized control structure of the DT method. The DSO employs price signals instead of direct command to control the energy planning of each aggregator, which makes it a decentralized control method. In the previous studies [7]-[9], an assumption was made that the parameters used by the DSO were the same as used by the aggregators. However, this might not be the case in practice and it may compromise the decentralized control concept by forcing the aggregators to report parameters to the DSO. Recognizing the importance of relaxing the link between the DSO and the aggregators, a different, but more practical, assumption is made in this paper, i.e. the DSO predicts the energy requirements of the flexible demands as the aggregators will not share this information with the DSO for protecting privacy of their customers. The prediction leads to a certain level of uncertainty of the DT method for congestion management. Secondly, there is uncertainty between the day-ahead energy planning and the real-time operation condition. For instance, the components of the power system or DER can fail with a certain level of probability. The forecast error of the energy production or consumption of the DER is another ex- 
ample.

By knowing the sources of the uncertainty and quantifying the uncertainty, it is possible to enhance the robustness of the DT method for congestion management through uncertainty management, which is the main contribution of this paper. The robustness is defined in this paper as the guarantee that the congestion probability is under a certain level with a given confidence level. Uncertainty management has been previously employed in other optimal scheduling problems in distribution networks, such as EV scheduling in [10] where a stochastic load profile is considered, and DG scheduling in [11] where stochastic productions of DERs are treated. However, unlike the DT method, both of them are using direct control methods without employing the DSO-Aggregator business model and the price incentives.

The paper is organized as follows. Section II presents the concept of the DT method for congestion management in a decentralized manner and the optimal energy planning through quadratic programing. The uncertainty sources of the decentralized control of the DT method are analyzed in section II. The robustness enhancement of the DT method through uncertainty management is proposed in section III. Two case studies regarding robustness enhancement are described and discussed in section IV followed by the conclusion.

\section{DT METHOD AND ITS UNCERTAINTY}

\section{A. Concept of DT for Congestion Management}

In [7], [8], the DT method to solve the congestion problem in a decentralized manner is proposed with the following procedure. Firstly, the DSO collects the flexible demand data, such as energy requirements and the availability, from the aggregators or by its own prediction. The DSO also needs the network information and the predicted spot price. Secondly, the DTs are calculated through the optimal energy planning by the DSO where the network constraints are respected. The DTs are sent to all the aggregators, together with the predicted energy prices as a reference to the aggregators. Thirdly, the aggregators make their own optimal plans independently with both the predicted spot prices and the received DTs. At last, the aggregators submit their energy plan/bids to the spot market. This DSO-Aggregator business model is still valid with the presence of parameter uncertainties of the optimal energy planning; however, the link between the DSO and the aggregators will be relaxed and the decentralized control architecture will be strengthened.

The concern regarding the regulatory issues related to the DT concept is addressed as follows. The DT collected by the DSO from the aggregators is similar to the congestion revenue collected by the independent system operator (ISO) in transmission networks. The concept of the congestion revenue at the transmission level is widely accepted in the electricity market with nodal price systems [12] or zonal price systems [13]. The congestion revenue will be used to improve the network conditions and reduce the congestions in the future, and benefits the parties who pay this congestion revenue. At the distribution level, the regulations in many European countries [14] require both cost reflectiveness, i.e. the network tariff reflects the cost incurred by different end-users, and non-discrimination. In many cases, the above two objectives are against to each other and a trade-off is made between them. If the non-discrimination has higher priority, the aggregators of flexible demands, who function as retailors or suppliers, can absorb the discrimination due to the DT and distribute the congestion cost among its customers. The aggregators optimize the consumptions at the cheapest hours and cause the potential congestions. Therefore, it is reasonable that the aggregators bear the cost of the congestion for rescheduling the flexible demands.

Moreover, the total revenue of the DSO is regulated by the regulators [14]. When the DT is considered in addition to the conventional flat tariff structure, the DT shall be included in the calculation of the total revenue of the DSO. The DSO uses DT as a tool to handle the potential congestions and shall not try to maximize the revenue through DT since the total revenue is regulated. In this sense, the DSO is independent in the DT concept while the aggregators are competitors.

\section{B. Quadratic Programming Based DT Method}

In [9], a quadratic programing formulation was proposed for the DT method. The DT method through quadratic programming can not only take into account the predicted energy price sensitivity of the spot market to the energy planning of flexible demands, but also offer a unique optimal solution of the energy planning at the aggregator side and therefore resolve the duel degeneracy issue of the optimization problem formulated through linear programming.

Taking EV as an example, the energy planning of flexible demands at the DSO side can be written as [9],

$$
\min _{p_{i, t}} \sum_{i \in N_{B}, t \in N_{T}} \frac{1}{2} p_{i, t}^{T} B_{i, t} p_{i, t}+\left(c_{t} \mathbf{1}\right)^{T} p_{i, t},
$$

subject to,

$$
\begin{gathered}
\sum_{i \in N_{B}} D E_{i}\left(p_{i, t}+q_{i, t}\right) \leq f_{t}, \forall t \in N_{T},\left(\lambda_{t}\right), \\
\sum_{\mathrm{t} \in N_{T}} p_{i, t} \geq d_{i}, \forall i \in N_{B}, \\
0 \leq p_{i, t} \leq a_{i, t} p_{i}^{\max }, \forall i \in N_{B}, t \in N_{T},
\end{gathered}
$$

where $B_{i, t} \in R^{m_{i} \times m_{i}}$ is matrix of the price sensitivity coefficient, $D \in R^{n_{L} \times n_{d}}$ is power transfer distribution factor (PTDF), $E_{i} \in R^{n_{d} \times m_{i}}$ is customer to load bus mapping matrix, $N_{B}$ is the set of aggregators, $N_{T}$ is the set of planning periods, $a_{i, t}$ is availability of EVs, $c_{t}$ is the baseline price, $d_{i} \in R^{m_{i}}$ is energy demands of EVs, $f_{t} \in R^{n_{t}}$ is line loading limit, $p_{i, t} \in R^{m_{i}}$ is charging power of EVs of one aggregator, $q_{i, t} \in R^{m_{i}}$ is inflexible demand, $p_{i}^{\max } \in R^{m_{i}}$ is the maximum charging power of EVs, $m_{i}$ is the number of customers of aggregator $i, n_{L}$ is the number of lines, $n_{d}$ is the number of buses, $\lambda_{t} \in R^{n_{L}}$ is Lagrange multiplier of the line loading limit constraint. 
In the energy planning, the line loading limit is ensured by constraint (2), energy demand requirement of EVs is fulfilled by constraint (3) and the allowed charging power of EVs is represented by (4).

The calculated DT, denoted as $r_{t}$, through the above optimization by the DSO, is equal to $D^{T} \lambda_{t}$, which will be sent to the aggregators, who then make the energy planning of the flexible demands on behalf of the owners. The energy planning of different aggregators is independent and without the information of the network constraints. Aggregator $i$ can employ the following optimization to make the energy planning [9].

$$
\min _{p_{i, t}} \sum_{t \in N_{T}} \frac{1}{2} p_{i, t}{ }^{T} B_{i, t} p_{i, t}+\left(c_{t} \mathbf{1}+E_{i}^{T} r_{t}\right)^{T} p_{i, t}
$$

subject to,

$$
\begin{gathered}
\sum_{\mathrm{t} \in \mathrm{N}_{t}} p_{i, t} \geq d_{i} \\
0 \leq p_{i, t} \leq a_{i, t}{ }^{T} p_{i}^{\max } \quad \forall t \in N_{T}
\end{gathered}
$$

In [9], it is proven that the convergence of the aggregator energy planning and the DSO one can be assured, i.e. the results and the line loading profiles resulting from the energy planning at the aggregator side will be same as those at the DSO side. As such, the DT method for the congestion management is realized in a decentralized manner. A strong assumption made by the authors was that the parameters of the optimization problem at the DSO side are same as those at the aggregator side. However, it is not necessarily true. In the following sub section, the source of parameter differences and the resulting uncertainty of the decentralized control of the DT method will be studied.

\section{Uncertainty of the Decentralized Control}

Table I gives one possible scenario of the information sources where the DSO and aggregators may have the required parameters ready for their energy planning respectively, which will be the assumption made for the study of the following sections. In this scenario, parameter $B_{i, t}$ is mainly to resolve the degeneracy issue in linear program and therefore the DSO should share it with the aggregators. Although the aggregators should have the freedom to choose their own energy price prediction resources, it is suggested that they (include DSO) all use the same source, since the DT method works through the total price (DT + energy price) as the price signal to control the behavior of the flexible demands. However, small discrepancy due to e.g. numerical errors of computers or modifications according to their own preferences is allowed as shown by case studies in [15]. The only uncertainty sources lie in the parameters $d_{i}$ and $a_{i, t}$, i.e. the driving patterns of EVs, where the DSO can only predict the driving pattern while the aggregators can have the detailed data from their customers. Since the study is focused on the difference between the DSO plan and the actual plans made by the aggregators that will be submitted to the day-ahead market, the data that the aggregators employ to determine the optimal energy plans will be deemed as accurate.

TABLE I

INFORMATION SOURCE OF DSO AND AGGREGATOR

\begin{tabular}{l|l|l}
\hline \multicolumn{1}{c|}{ Para. } & \multicolumn{1}{|c}{ DSO } & \multicolumn{1}{c}{ Aggregator } \\
\hline$B_{i, t}$ & Predicted by DSO & From DSO \\
\hline$c_{t}$ & $\begin{array}{l}\text { Predicted by DSO or } \\
\text { third party }\end{array}$ & $\begin{array}{l}\text { From DSO or choose the same } \\
\text { third party as the DSO do }\end{array}$ \\
\hline$D, f_{t}$ & Determined by DSO & Not needed \\
\hline$E_{i}, p_{i}^{\max }$ & $\begin{array}{l}\text { From customers' } \\
\text { subscription }\end{array}$ & From customers' subscription \\
\hline$d_{i}, a_{i, t}$ & Predicted by DSO & From the customers' reports \\
\hline
\end{tabular}

It is important to know the distribution of the prediction error of predicted parameters $d_{i}$ and $a_{i, t}$ in order to analyze the accuracy of the DSO's energy plan (compared to the energy plans of the aggregators) and then enhance the robustness of the DT method. In [16], the driving pattern (driving distance and availability) of a large number of drivers has been surveyed. The study is based on Denmark and it can help illustrate the discussion of parameter randomness in this section and the methodology of robust enhancement in the following sections without affecting the generality of the discussion.

In Denmark, a large portion of the drivers (about $40 \%$ on weekdays, about $50 \%$ on weekends) do not drive at all according to the daily observations (interviews). The percentage of the drivers who drive on a particular day diminishes as the driving distance goes up. The average driving distance is about $30 \mathrm{~km}$ while it varies from weekdays to weekends. From the statistics point of view, the prediction error (parameter used by the DSO minus the one used by the aggregator) of the predicted driving distance of each EV can be considered to have a normal distribution with variance $\sigma^{2}$ as shown in Fig. 1. The chance of the error bigger (or less) than a predefined number can be calculated from the normal distribution table, e.g. the probability of the error that is positive and bigger than $2 \sigma$ is only $2.5 \%$ (the shadow part of Fig. 1).

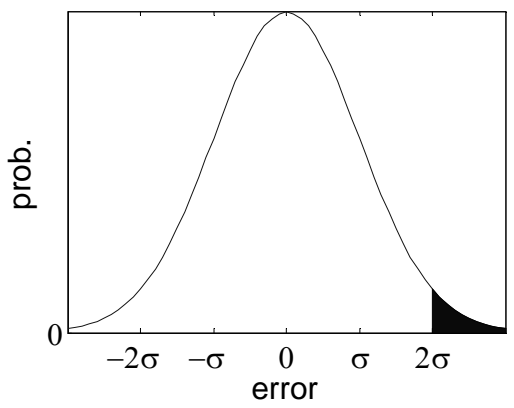

Fig. 1. Normal distribution of the prediction error

The energy demand of an EV has a linear relationship with the driving distance, which is defined as the parameter "energy consumption per km" (Table II). Therefore, the prediction error of the energy demands $d_{i}$ also has a normal distribution. 
The parameter $a_{i, t}$ is also influenced by the randomness of the driving pattern. The availability of an EV can be modeled through two random variables, namely arriving (home) time and leaving (home) time. For example, in Fig. 2, $y$ is the arriving time while $x$ is the leaving time. The availability $a_{i, t}$ ( $j$-th element) can be determined by the following rules.

$$
\left\{a_{i, t}\right\}_{j}=\left\{\begin{array}{lc}
1 & t \leq\lfloor x\rfloor, \text { or }, t \geq\lceil y\rceil \\
0 & \lceil x\rceil \leq t \leq\lfloor y\rfloor \\
x-\lfloor x\rfloor & t=\lfloor x\rfloor, t \neq\lfloor y\rfloor \\
\lceil y\rceil-y & t=\lfloor y\rfloor, t \neq\lfloor x\rfloor \\
x-\lfloor x\rfloor+\lceil y\rceil-y & t=\lfloor x\rfloor=\lfloor y\rfloor
\end{array} \quad, t \in N_{T}\right.
$$

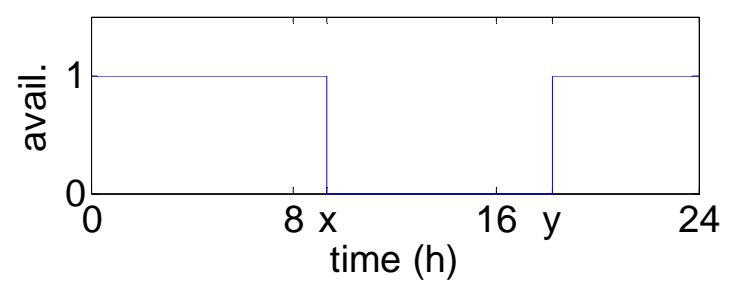

Fig. 2. Illustration of the modeling of the availability ( $x$ is the leaving time and $y$ is the arriving time)

The prediction error of the predicted arriving time has a normal distribution, so does the prediction error of the predicted leaving time.

\section{RoBustness ENHANCEMENT OF THE DT MethoD THROUGH UNCERTAINTY MANAGEMENT}

The criterion of the robustness enhancement for the DT method is defined as: The probability of the line overloading $\varepsilon$ (percentage) is no bigger than a predefined number, e.g. $1-\eta$ with $\eta$ being a confidence level, under the uncertainty of the parameters. The task of the robustness enhancement is to find such DT that the above criterion is fulfilled.

\section{A. Problem Formulation}

According to the concept described in section II.A, DT should be able to serve as a proper price signal such that the optimal energy plans made by the aggregators based on the received DT will result no (or alleviated) congestion on the distribution network. The method to determine such DT described in section II.B, i.e. (1)-(4), is based on a deterministic optimization, which is not suitable for determining a DT with uncertainty constraints presented, because of the following two facts: 1) The Lagrange multiplier of uncertainty constraints is very difficult to find; 2) The Lagrange multiplier with uncertainty constraints presented is no long the marginal price of the congestion cost.

A method that can determine the DT without using Lagrange multiplier can be written as a two-level optimization,

$$
\min _{\mathrm{r}_{\mathrm{t}}} \sum_{t \in N_{T}}\left\|r_{t}\right\|_{1}
$$

subject to,

$$
\begin{aligned}
& \sum_{i \in N_{B}} D E_{i}(\left.p_{i, t}+q_{i, t}\right) \leq f_{t}, \forall t \in N_{T}, \\
& r_{t} \geq 0, \forall t \in N_{T}, \\
& p_{i, t} \in \arg \min \{(5):(6)-(7)\}, \forall i \in N_{B} .
\end{aligned}
$$

In the above two-level optimization, variable $p_{i, t}$ is the unique minimizer of the inner optimization, which is a strictly convex optimization. The parameters $d_{i}$ and $a_{i, t}$ in (6)-(7) are deemed as random parameters; therefore constraint (9) cannot be fulfilled with certainty. A chance constrained two-level optimization can be formed as (9) subject to,

$$
\text { prob. }\left\{\sum_{i \in N_{B}} D E_{i}\left(p_{i, t}+q_{i, t}\right) \leq(1+\varepsilon) f_{t}, \forall t \in N_{T}\right\} \geq \eta,
$$

and (11)-(12), where $\eta$ is the confidence level and has a typical value of $0.9,0.95$ or 0.99 .

The above chance constrained two-level optimization is very difficult to solve directly. An alternative method is developed in this paper to solve it, i.e. an iteration method based on the probability analysis and the knowledge gained in our previous work, i.e. the deterministic method to determine DT [9] and sensitivity analysis [15]. The main idea of the iteration method is explained as follows.

Firstly, the chance constraint (13) is relaxed to be deterministic. Meanwhile, the parameter $\varepsilon$ becomes a control variable which will take different value in each iteration step according to the results of the previous iteration step. Random parameters $d_{i}$ and $a_{i, t}$ are replaced with their expected values, i.e. $\bar{d}_{i}$ and $\bar{a}_{i, t}$, respectively.

Secondly, the two-level optimization is reformed as the standard form descripted in section II.B (the one-level optimization at the DSO side) in order to determine an iterative DT, denoted as $r_{t}^{(k)}$, based on the theory proven in [9]: The solution at the aggregator side ((5)-(7)) is the same as the solution at the DSO side ((1)-(4)) if $r_{t}=D^{T} \lambda_{t}$ is hold. Thus, the optimization to determine the iterative DT can be written as (1) subject to,

$$
\begin{gathered}
\sum_{i \in N_{B}} D E_{i}\left(p_{i, t}+q_{i, t}\right) \leq\left(1+\varepsilon_{t}^{(k)}\right) f_{t}, \forall t \in N_{T},\left(\lambda_{t}^{(k)}\right), \\
\sum_{t \in N_{T}} p_{i, t} \geq \bar{d}_{i}, \forall i \in N_{B}, \\
0 \leq p_{i, t} \leq \bar{a}_{i, t} p_{i}^{\max }, \forall i \in N_{B}, t \in N_{T},
\end{gathered}
$$

where $k$ represents the iteration step.

Thirdly, the chance constraint (13) will be verified through probability calculation based on the uncertainty analysis described in section II.C and the sensitivity analysis of the optimization (5)-(7) with updated DT, i.e. $r_{t}^{(k)}=D^{T} \lambda_{t}^{(k)}$, which will be elaborated in section III.B. If (13) is fulfilled, then we find the final DT; otherwise the control parameter $\varepsilon_{t}^{(k)}$ will be updated and perform the next iteration. The detailed procedure of the iteration method will be described in section III.C.

\section{B. Probability Calculation}

Probability calculation is an important step in the uncertainty management. It is carried out through uncertainty anal- 
ysis of the parameters and the sensitivity analysis of the optimal solution of the energy planning at the aggregator side.

1) Sensitivity analysis:

The sensitivity analysis is to determine the changes of the optimal solution due to the changes of the parameters and it shall be done per optimal solution [15]. The optimization (5) -(7) (the optimization at the aggregator side) can be rewritten with updated parameters as,

$$
\min _{p_{i, t}} \sum_{t \in N_{T}} \frac{1}{2} p_{i, t}{ }^{T} B_{i, t} p_{i, t}+\left(c_{t} 1+E_{i}^{T} r_{t}^{(k)}\right)^{T} p_{i, t}
$$

subject to,

$$
\begin{gathered}
\sum_{\mathrm{t} \in \mathrm{N}_{T}} p_{i, t} \geq \bar{d}_{i}, \\
0 \leq p_{i, t} \leq \bar{a}_{i, t}{ }^{T} p_{i}^{\max } \quad \forall t \in N_{T} .
\end{gathered}
$$

Assume that the optimal solution of the above deterministic optimization problem is $\left(p_{i, 1}^{*}, p_{i, 2}^{*}, p_{i, 3}^{*}, \ldots, p_{i, n_{T}}^{*}\right)^{(k)}$, which should be also the optimal solution of (1) subject to (14)-(16). Now assume that the parameters $d_{i}$ and $a_{i, t}$ at the right side of (18)-(19) can vary according to their statistic distributions with their centers $\bar{d}_{i}$ and $\bar{a}_{i, t}$ respectively, note that $d_{i}=\bar{d}_{i}+\Delta d_{i}$ and $a_{i, t}=\bar{a}_{i, t}+\Delta a_{i, t}$. It therefore can be determined how the optimal solution will change according to the changes of the parameters $d_{i}$ and $a_{i, t}$, i.e. $\Delta d_{i}$ and $\Delta a_{i, t}$, near the optimal point $\left(p_{i, 1}^{*}, p_{i, 2}^{*}, p_{i, 3}^{*}, \ldots, p_{i, n_{T}}^{*}\right)^{(k)}$ through the sensitivity analysis method presented in [15].

According to [15], the optimization problem (17)-(19) should be rewritten as a standard form as below ( $i$ is fixed) with inactive constraints removed,

$$
\min _{x} \frac{1}{2} p^{T} B x+g^{T} p
$$

subject to,

$$
A p=b
$$

where $p$ is $\left[p_{i, 1}{ }^{T}, p_{i, 2}{ }^{T}, p_{i, 3}{ }^{T}, \ldots, p_{i, n_{T}}{ }^{T}\right]^{T}$,

$B$ is $\left[\begin{array}{ccccc}B_{i, 1} & & & & \\ & B_{i, 2} & & & \\ & & B_{i, 3} & & \\ & & & \cdots & \\ & & & & B_{i, n_{T}}\end{array}\right]$,

$g$ is $\left[\begin{array}{l}c_{1} 1+E_{i}^{T} r_{1}^{(k)} \\ c_{2} 1+E_{i}^{T} r_{2}^{(k)} \\ c_{3} 1+E_{i}^{T} r_{3}^{(k)} \\ \cdots \\ c_{n_{T}} 1+E_{i}^{T} r_{n_{T}}^{(k)}\end{array}\right]^{T}$,

$A$ and $b$ are coefficients of the active constraints in (18)-(19).

The optimal solution changes over the changes of the vector $b$ can be obtained as (22).

$$
\frac{\partial p}{\partial b}=B^{-1} A^{T}\left(A B^{-1} A^{T}\right)^{-1}
$$

The line loading change at a particular hour $t$ is the summation of the changes of the EV charging power $p$, which can be written as,

$$
\sum_{i \in N_{B}} D E_{i}\left(\Delta p_{i, t}\right)=\sum_{i \in N_{B}} D E_{i} \frac{\partial p_{i, t}}{\partial b} \Delta b
$$

where $\frac{\partial p_{i, t}}{\partial b}$ can be retrieved from matrix $\frac{\partial p}{\partial b}$ by taking the corresponding rows.

2) Chance of overloading due to energy demand:

Since the change of the energy demand $d_{i}$ results the change of $b$ vector of (21), the sensitivity of the change of the line loading over the change of the energy demand can be calculated through (22) and (23). Moreover, the probability of a predefined overloading $\varepsilon$, e.g. line overloading $5 \%$ (5\% more than the line loading limit), due to the prediction error of the energy demand, denoted as $\Delta d_{i}$, can be calculated through combining the statistic distribution of the prediction error into the calculation. Specifically, the probability can be determined by,

$$
\rho_{d}=\text { prob. }\left\{\sum_{i \in N_{B}} \alpha_{i}^{T} \Delta d_{i} \geq \varepsilon f_{t}\right\},
$$

where $\alpha_{i}$ is the sensitivity coefficients corresponding to $\Delta d_{i}$ and can be retrieved from (23).

3) Chance of overloading due to availability:

Similarly, the prediction error of the availability $a_{i, t}$ can be determined through (8) and the changes of the line loading can be determined through (22) and (23). The probability of a predefined overloading $\varepsilon$ due to the prediction error of the availability, denoted as $\Delta a_{i, t}$, can be calculated by,

$$
\rho_{a}=\operatorname{prob} .\left\{\sum_{i \in N_{B}, t \in N_{T}} \beta_{i, t}{ }^{T} \Delta a_{i, t} \geq \varepsilon f_{t}\right\},
$$

where $\beta_{i, t}$ is the sensitivity coefficient corresponding to $\Delta a_{i, t}$ and can be retrieved from (23).

Combining (24) and (25), the probability of a predefined overloading $\varepsilon$ due to $\mathrm{EV}$ parameters can be obtained as,

$$
\rho=\text { prob. }\left\{\sum_{i \in N_{B}} \alpha_{i}^{T} \Delta d_{i}+\sum_{i \in N_{B}, t \in N_{T}} \beta_{i, t}{ }^{T} \Delta a_{i, t} \geq \varepsilon f_{t}\right\} .
$$

The distribution $\sum_{i \in N_{B}} \alpha_{i}^{T} \Delta d_{i}+\sum_{i \in N_{B}, t \in N_{T}} \beta_{i, t}{ }^{T} \Delta a_{i, t}$, denoted by $F$, is a normal distribution since each element of $\Delta d_{i}$ and $\Delta a_{i, t}$ is a normal distributed random variable and $F$ is a linear combination of them. It is assumed that $\Delta d_{i}$ and $\Delta a_{i, t}$ are independent random variables since the mathematical complexity incurred from the dependency of these random variables is not a focus of this paper. The variance of the joint distribution $F$ is a linear combination of the variance of each random variable. 


\section{Procedure of Robustness Enhancement}

In order to achieve robustness enhancement, a procedure is designed and illustrated in Fig. 3. The procedure that the DSO should follow is:

1) Forecast the day-ahead energy prices.

2) Forecast the driving behaviors of EVs, including driving distance, arriving time and leaving time.

3) Set the initial line loading limit to be $100 \%$, i.e. $\varepsilon_{t}^{(0)}=0$ in (14).

4) Perform the optimal energy planning at the DSO side through QP and calculate the DT, i.e. solve (1) subject to (14)-(16).

5) Perform the sensitivity analysis of the energy planning at the aggregator side, i.e. (17)-(19). Determine $\alpha_{i}$ and $\beta_{i, t}$ in (26).

6) Perform the probability calculation, i.e. determine the value $\rho$ of (26).

7) Check whether the probability $\rho$ is no bigger than $1-\eta$. If yes, publish the final DT and terminate the algorithm; otherwise,

8) Calculate the new line loading limit, which is achieved by pushing down the limit with a small step. The step size can be a fixed small number, e.g. $0.5 \%$, or estimated by the Newton-Raphson method noticing the fact that the sensitivity matrix is the Jacobian matrix.

9) Go back to step 4.

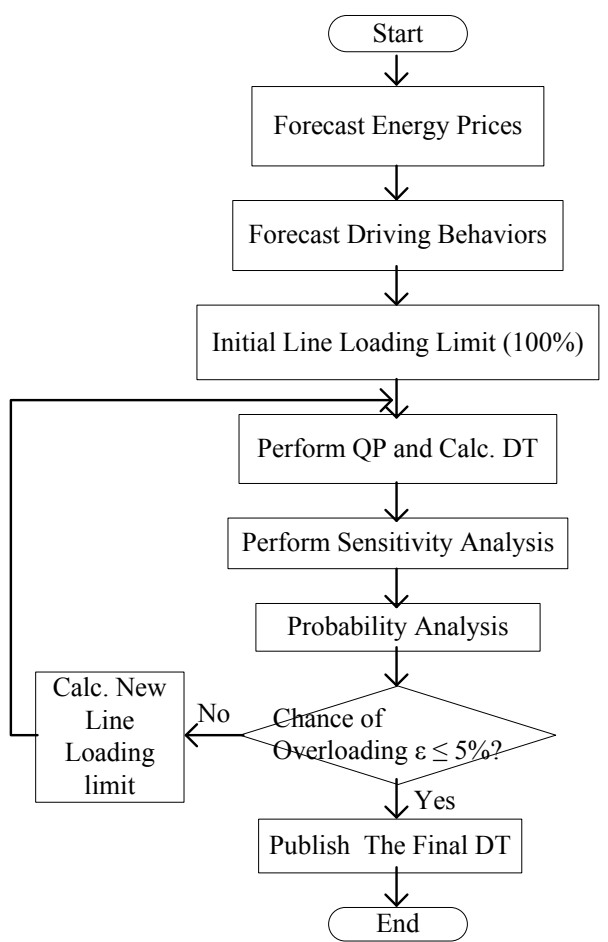

Fig. 3. Flowchart illustrating the procedure of robustness enhancement

\section{CASE STUdies}

Case studies were conducted using the Danish driving pattern and the Bus 4 distribution system of the Roy Billinton Test
System (RBTS) [17] to demonstrate the robustness enhancement of the DT method through uncertainty management. The details of the case studies are presented in this section.

\section{A. Grid Data}

The single line diagram of the Bus 4 distribution network is shown in Fig. 4. Line segments of the feeder one are labeled in Fig. 4, among which L2, L4, L6, L8, L9, L11, and L12 refer to the transformers connecting the corresponding load points (LP1 to LP7). The study is focused on this feeder because it has the most diversity among all the feeders: 5 residential load points with different peak conventional demands and two commercial load points. Each of the residential load points (LP1-5) has 200 customers while each of the commercial load points (LP6-7) has 10 customers. The peak conventional demands of residential customers are assumed to occur at 18:00 when people come home and start cooking (shown in Fig. 7).

The key parameters of the simulations are listed in Table II The EV availability shown in Fig. 5 is from the driving pattern study in [16]. Assume that there are two aggregators (agg1 and agg2); one has 40 customers per load point and the other has 160 customers per load point.

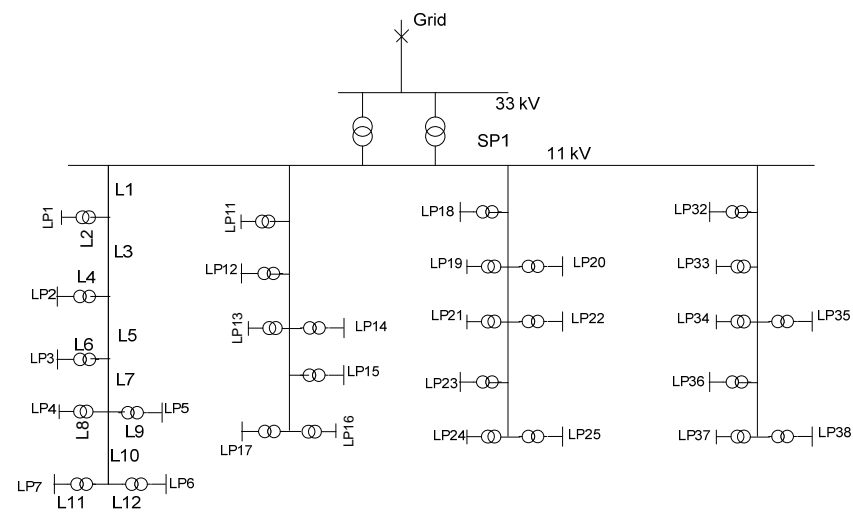

Fig. 4. Single line diagram of the distribution network

TABLE II

Key PARAMETERS FOR THE SIMULATION

\begin{tabular}{ll}
\hline Parameter & value \\
\hline EV battery size & $25 \mathrm{kWh}$ \\
\hline Peak charging power & $11 \mathrm{~kW}(3$ phase $)$ \\
\hline Energy consumption per km, $\phi$ & $150 \mathrm{Wh} / \mathrm{km}$ \\
\hline expected driving distance & $40 \mathrm{~km}$ \\
\hline expected leaving time & Hour 8 \\
\hline expected arriving time & Hour 18 \\
\hline$\sigma$ of predicted driving distance, $\sigma_{d}$ & $20 \mathrm{~km}$ \\
\hline$\sigma$ of predicted leaving time & 60 minute \\
\hline$\sigma$ of predicted arriving time & 60 minute \\
\hline Line loading limit: L2 & $1400 \mathrm{~kW}$ \\
\hline Line loading limit: L3 & $6000 \mathrm{~kW}$ \\
\hline Line loading limit: L4 & $1700 \mathrm{~kW}$ \\
\hline
\end{tabular}




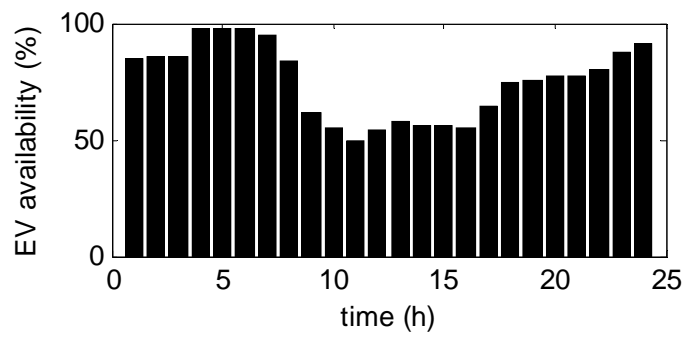

Fig. 5. EV availability

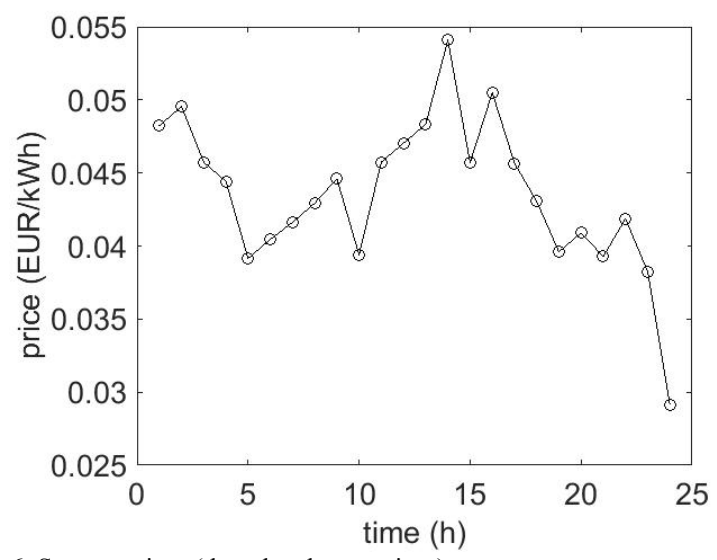

Fig. 6. System prices (day-ahead spot prices)

\section{B. Results of The Robustness Enhancement}

\section{1) Case One:}

According to the procedures proposed in section III.C, the basic energy prices are firstly predicted and the results are shown in Fig. 6. In this sub section, the prediction of the energy price is assumed to be perfect, i.e. the aggregators and the DSO use the same prediction. Then the driving behaviors including driving distance, arriving time and leaving time are predicted as list in Table II. The energy planning at the DSO side is performed with initial values, i.e. $\varepsilon_{t}^{(0)}=0$ in (14), and the results are plotted in Fig. 7.
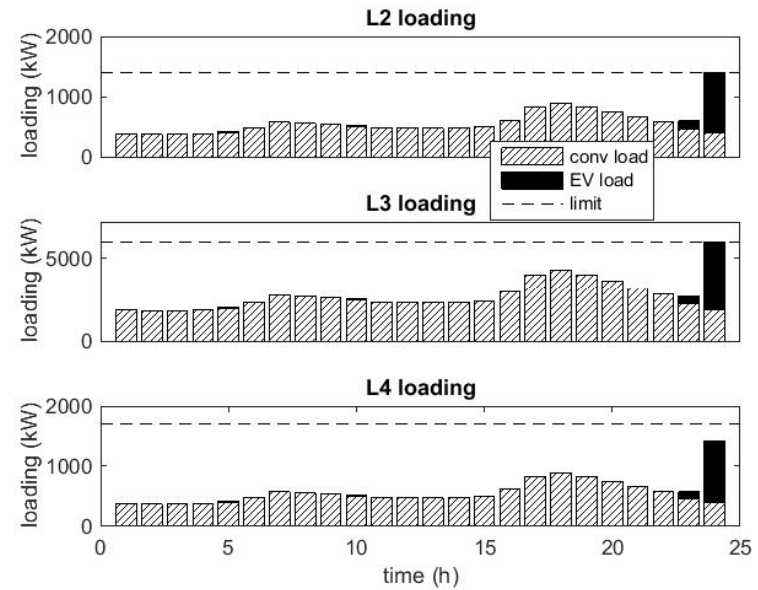

Fig. 7. Line loading of the initial energy planning at DSO side

Then the sensitivity analysis of the line loading change to the change of energy demand, arriving time and leaving time is performed at the optimal point. Part of the results (only regarding L3) is shown in Table III. It can be seen that the line loadings (L3) at hour 23 and 24 are sensitive to energy demand of EVs (the results are synthesized from the individual sensitivity of each EV, because it is not necessary to show the results of a thousand EVs), while it is less sensitive at hour 5, 6, 10 and 19. The change of energy demand will not affect the line loadings at other hours since the sensitivity coefficient is zero. For the change of the arriving time, it will only influence the line loading at hour 24 and the influence is insignificant (one minute change of the arriving time will only lead to $3.2 \mathrm{~kW}$ change of the line loading). The reason can be explained by the fact that the expected arriving time (see Table II) is far away from the congestion hour, namely hour 24 . The same reason explains that the change of the leaving time has insignificant influence to the line loading change.

TABLE III

SENSITIVITY OF L3 LoAdING CHANGE TO THE CHANGE of ENERGY DEMAND, ARRIVING TIME AND LEAVING TIME; DATA OF OTHER HOURS IS ZERO.

\begin{tabular}{|l|l|l|l|}
\hline Hour & $\begin{array}{l}\text { Energy Demand } \\
\text { (kW/kWh) }\end{array}$ & $\begin{array}{l}\text { Arriving Time } \\
\text { (kW/min) }\end{array}$ & $\begin{array}{l}\text { Leaving Time } \\
\text { (kW/min) }\end{array}$ \\
\hline $\mathbf{5}$ & 32 & 0 & 0 \\
\hline $\mathbf{6}$ & 8 & 0 & 0 \\
\hline $\mathbf{1 0}$ & 16 & 0 & 0 \\
\hline $\mathbf{1 9}$ & 8 & 0 & 0 \\
\hline $\mathbf{2 3}$ & 352 & 0 & 0 \\
\hline $\mathbf{2 4}$ & 384 & 3.2 & 0 \\
\hline
\end{tabular}

The next step is to carry out the probability analysis. Take L3 as an example (both L2 and L3 are critical which can be observed from Fig. 7; however L2 can be analyzed through the same method as for L3). The line loading limit is $6000 \mathrm{~kW}$ and the probability of the overloading $5 \%$, i.e. $5 \%$ of the original limit or $300 \mathrm{~kW}$, will be estimated. The critical hour (congestion hour) is hour 24 and the sensitivities associated with arriving time and leaving time are negligible according to Table IV. The standard deviation of $F$ can be determined as $\sqrt{\alpha_{1}^{T} \alpha_{1}+\alpha_{2}{ }^{T} \alpha_{2}} \phi \sigma_{d}=43.26(\mathrm{~kW})$. Therefore, the probability of $F$ bigger than $300 \mathrm{~kW}$ is very low (less than $0.1 \%$ ). According the procedure, the algorithm can be terminated with the final DT (the $1^{\text {st }}$ iteration column of Table IV).

2) Case Two:

However, if the DSO is more conservative, e.g. the DSO set its goal to be that the probability of overloading $(0 \%)$ is no bigger than $5 \%$, the algorithm needs to continue to the second iteration because after the first iteration, the probability of overloading is $50 \%$. The new line loading limit of L3 at the critical hour is reduced by $0.5 \%$ (a fixed small step), i.e. the new line loading limit is $5970 \mathrm{~kW}$. The procedure is repeated from step 4 . The sensitivity and the standard deviation of $F$ are almost not changed from the first iteration. The probability of $-30+F \geq 0$ (noted that the line loading limit is reduced by 30 $\mathrm{kW}$ in the optimal energy planning) is $24.5 \%$. The algorithm needs to continue to the third and fourth iteration. After the fourth iteration, the probability is reduced to $2.1 \%$ and the 
algorithm can be terminated with the final DT (the $4^{\text {th }}$ iteration column of Table IV).

The results of Case One and Case Two are listed in Table IV. As the L3 loading limitation sets to be lower and lower in the optimal energy planning at the DSO side, the DT at LP2-5 goes up gradually (DT at LP1 is not changed because the enhancement regarding L2 overloading has not been carried out yet; it can be done by following the same procedure for L3), which is reasonable because it needs to shift more power consumption to other hours. It can be seen from the final energy planning results shown in Fig. 8 that $90 \mathrm{~kW}$ is shifted from hour 24 to hour 23 comparing to the original planning (Fig. 7). The benefit is that the probability of L3 overloading (the physical limitation is still $6000 \mathrm{~kW}$ ) is only $2.1 \%$, which is the confidence that the DSO has on the DT method for congestion management.
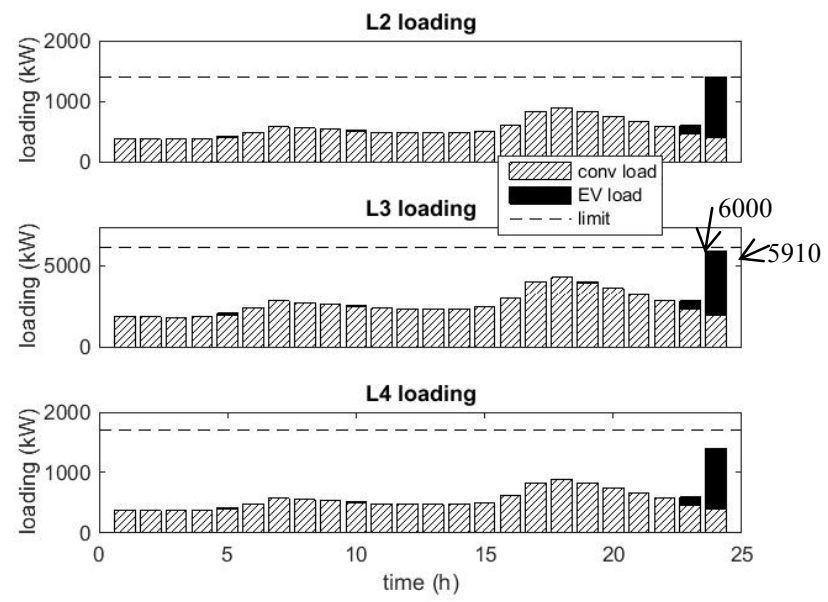

Fig. 8. The final energy planning results at the DSO side

TABLE IV

RESULTS OF EACH ITERATION

\begin{tabular}{|l|l|l|l|l|}
\hline & $\mathbf{1}^{\text {st }}$ iter & $\mathbf{2}^{\text {nd }}$ iter & $\mathbf{3}^{\text {rd }}$ iter & $\mathbf{4}^{\text {th }}$ iter \\
\hline $\begin{array}{l}\text { DT at LP1, } \\
\text { Hour 24 } \\
\text { (DKK/kWh) }\end{array}$ & 0.0519 & 0.0519 & 0.0519 & 0.0519 \\
\hline $\begin{array}{l}\text { DT at LP2-5, } \\
\text { Hour 24 } \\
\text { (DKK/kWh) }\end{array}$ & 0.0511 & 0.0514 & 0.0518 & 0.0521 \\
\hline $\begin{array}{l}\text { Prob. of over- } \\
\text { loading 5\% }\end{array}$ & $<0.1 \%$ & - & - & - \\
\hline $\begin{array}{l}\text { Prob. of over- } \\
\text { loading }\end{array}$ & $50 \%$ & $24.5 \%$ & $8.2 \%$ & $2.1 \%$ \\
\hline $\begin{array}{l}\text { L3 loading } \\
\text { limitation (kW) }\end{array}$ & 6000 & 5970 & 5940 & 5910 \\
\hline
\end{tabular}

\section{CONCLUSIONS}

The paper presents the uncertainty management of the DT method with the presence of stochastic parameters of the flexible demands. The uncertainty comes from the fact that the DSO needs to forecast the behavior and energy requirement of the flexible demands since the DT method has a decentralized control architecture, where the aggregators make day-ahead energy plans independently without considering network con- straints. With the uncertainty management, the robustness of the DT method is enhanced that the probability of congestion (or an allowed congestion level) resulting from the DT method is under a predefined level.

The main limitation of the proposed uncertainty management method lies on the sensitivity analysis carried out in each iteration step, which has an assumption that the changes of the parameters are reasonably small. Therefore, the deviations of the forecast errors should not be too big; otherwise, the accuracy of the sensitivity analysis will be compromised.

In the future work, the uncertainty regarding the forecasted conventional loads can be included, which should be more straightforward than handling the uncertainty of the energy requirement of the flexible demands. Further, a combination of the DT method and intra-day (or real-time) congestion management methods can be developed, which can offer the DSO more choices on risk management and cost of the congestion management in the distribution network.

\section{REFERENCES}

[1] A. R. Malekpour, A. R. Seifi, M. R. Hesamzadeh, and N. Hosseinzadeh,"An optimal load shedding approach for distribution networks with DGs considering capacity deficiency modelling of bulked power supply," in Proc. Power Engineering Conference, pp. 1-7.

[2] M. Alonso, H. Amarís, C. Álvarez, and R. Albarracín, "Reactive power planning in distribution networks with distributed generation," in Proc. 7th Mediterranean Conference and Exhibition on Power Generation, Transmission, Distribution and Energy Conversion (MedPower 2010), pp. $1-7$.

[3] R. A. Verzijlbergh, L. J. De Vries, and Z. Lukszo,"Renewable energy sources and responsive demand. Do we need congestion management in the distribution grid?," IEEE Trans. Power Syst., pp. 1-10, 2014.

[4] P. Bach Andersen, J. Hu, and K. Heussen, “Coordination strategies for distribution grid congestion management in a multi-actor, multi-objective setting," in Proc. 3rd IEEE PES Innovative Smart Grid Technologies Europe (ISGT Europe), pp. 1-8.

[5] P. M. Sotkiewicz and J. M. Vignolo,"Nodal Pricing for Distribution Networks: Efficient Pricing for Efficiency Enhancing DG," IEEE Trans. Power Syst., vol.21, no.2, pp. 1013-1014, May 2006.

[6] G. T. Heydt, B. H. Chowdhury, M. L. Crow, D. Haughton, B. D. Kiefer, F. Meng, and B. R. Sathyanarayana,"Pricing and control in the next generation power distribution system," IEEE Trans. Smart Grid, vol.3, no.2, pp. 907-914, Jun. 2012.

[7] N. O'Connell, Q. Wu, J. Østergaard, A. H. Nielsen, S. T. Cha, and Y. Ding, "Day-ahead tariffs for the alleviation of distribution grid congestion from electric vehicles," Electr. Power Syst. Res., vol.92, pp. 106-114, 2012.

[8] R. Li, Q. Wu, and S. S. Oren,"Distribution locational marginal pricing for optimal electric vehicle charging management," IEEE Trans. Power Syst., vol.29, no.1, pp. 203-211, Jan. 2014

[9] S. Huang, Q. Wu, S. S. Oren, R. Li, and Z. Liu, “Distribution Locational Marginal Pricing Through Quadratic Programming for Congestion Management in Distribution Networks," IEEE Trans. Power Syst., vol.30, no.4, pp. 2170-2178, Jul. 2015.

[10] K. Clement-Nyns, E. Haesen, and J. Driesen,"The Impact of Charging Plug-In Hybrid Electric Vehicles on a Residential Distribution Grid," IEEE Trans. Power Syst., vol.25, no.1, pp. 371-380, Feb. 2010.

[11] Y. Cao, Y. Tan, C. Li, and C. Rehtanz,“Chance-Constrained Optimization-Based Unbalanced Optimal Power Flow for Radial Distribution Networks," IEEE Trans. Power Deliv., vol.28, no.3, pp. 1855-1864, Jul. 2013

[12] D. Kirschen and G. Strbac,Fundamentals of Power System Economics. England: John Wiley \& Sons Ltd, 2004.

[13] R. Djabali, J. Hoeksema, and Y. Langer. (2011). COSMOS description CWE Market Coupling algorithm. [online]. Available: https://www.apxgroup.com/wp-content/uploads/COSMOS_public_descr iption2.pdf. 
[14] EURELECTRIC. Network tariff structure for a smart energy system. [online]. Available:

http://www.eurelectric.org/media/80239/20130409_network-tariffs-pape $r$ final to publish-2013-030-0409-01-e.pdf.

[15] S. Huang, Q. Wu, Z. Liu, and H. Zhao, "Sensitivity analysis of dynamic tariff method for congestion management in distribution networks," in Proc. 2015 IEEE PES General Meeting, accepted.

[16] Q. Wu, A. H. Nielsen, J. Østergaard, F. Marra, and C. Træholt, “Driving pattern analysis for electric vehicle (EV) grid integration study," in Proc. 2010 IEEE PES Innovative Smart Grid Technologies Conference Europe (ISGT Europe), pp. 1-6.

[17] R. N. Allan, R. Billinton, I. Sjarief, L. Goel, and K. S. So,“A reliability test system for educational purposes-basic distribution system data and results," IEEE Trans. Power Syst., vol.6, no.2, pp. 813-820, May 1991. 